\title{
A rapid response team is associated with reduced overall hospital mortality in a Chinese tertiary hospital: a 9-year cohort study
}

\author{
Xiao-Yan Gong ${ }^{1 \#}$, Yong-Gang Wang ${ }^{1 \#}$, Hong-Yi Shao ${ }^{2}$, Peng Lan ${ }^{1}$, Ru-Shuang Yan $^{1}$, Kong-Han Pan ${ }^{1}$, \\ Jian-Cang Zhou ${ }^{1}$ \\ ${ }^{1}$ Department of Critical Care Medicine, Sir Run Run Shaw Hospital, Zhejiang University School of Medicine, Hangzhou 310016, China; \\ ${ }^{2}$ Department of Emergency Intensive Care Medicine, Affiliated Central Hospital, Shaoxing University, Shaoxing 312030, China \\ Contributions: (I) Conception and design: XY Gong, JC Zhou; (II) Administrative support: KH Pan; (III) Provision of study materials or patients: \\ YG Wang; (IV) Collection and assembly of data: XY Gong, HY Shao, JC Zhou; (V) Data analysis and interpretation: P Lan, RS Yan, JC Zhou; (VI) \\ Manuscript writing: All authors; (VII) Final approval of manuscript: All authors. \\ \#These authors contributed equally to this work. \\ Correspondence to: Kong-Han Pan; Jian-Cang Zhou. Department of Critical Care Medicine, Sir Run Run Shaw Hospital, No. 3 Qing Chun Road \\ East, Hangzhou 310016, China. Email: pankonghan@zju.edu.cn; jiancangzhou@zju.edu.cn.
}

\begin{abstract}
Background: Although the evidence for its effectiveness remains uncertainty, rapid response systems are implemented across many hospitals across the world. Increasingly, hospitals in China have recently started to adopt a medical emergency or rapid response team (RRT). Hence, we aimed to determine whether the implementation of an RRT in Chinese hospitals also improved outcomes.

Methods: Our hospital is a Joint Commission International (JCI) accredited, tertiary teaching hospital with 1,200 beds. We conducted a retrospective cohort study comparing 60 months after the implementation of the RRT (January 1, 2013, to December 31, 2017) and 36 months before implementation (January 1, 2009, to December 31, 2011). The outcomes included the overall hospital mortality and incidence of codes.

Results: We analyzed 144,673 non-obstetric hospital admissions and 1,269,621 patient days in the control period and 348,687 non-obstetric hospital admissions and 2,361,913 patient days after the RRT implementation. The RRT was activated 834 times (2.39 calls per 1,000 patients and 0.35 call per 1,000 patient-days). There was no difference in the code rate (0.23 vs. 0.17 per 1,000 patient days, $\mathrm{P}=0.379$ ) between the two periods. Although the hospital mortality had remained stable around 3.0 per 1,000 patients from 2009 to 2011, there was a significant 40\% decrease of overall hospital mortality from 2.95 to 1.77 per 1,000 non-obstetric patients after the implementation of RRT $(\mathrm{P}=0.001)$, and the annual mortality showed a consistent decrease $(\mathrm{P}=0.037$ for the trend). Moreover, the increase of RRT activations was significantly correlated with the decrease of hospital mortality $(\mathrm{P}=0.025)$.
\end{abstract}

Conclusions: RRT implementation was associated with reduced overall hospital mortality in a Chinese tertiary hospital.

Keywords: Rapid response team; medical emergency team; hospital mortality; cardiac arrest

Submitted Dec 16, 2019. Accepted for publication Feb 06, 2020.

doi: $10.21037 /$ atm.2020.02.147

View this article at: http://dx.doi.org/10.21037/atm.2020.02.147

\section{Introduction}

Studies have suggested that $10 \%$ of hospitalized patients suffered serious adverse events, and up to $25 \%$ of them result in death or permanent disability $(1,2)$. In the United States, an estimated 290,000 in-hospital cardiac arrests occur each year (3). Among them, clinical deterioration is common before in-hospital cardiac arrest, while $60 \%$ have at least 1 abnormal vital sign $1-4 \mathrm{~h}$ before the cardiac arrest, and $13.4 \%$ have at least 1 severely abnormal sign (4). Therefore, it is an ideal clinical strategy to detect early 
and implement rapid intervention strategies during this short "window" period to prevent subsequent cardiac arrest and save lives. A rapid response system consists of a multidisciplinary team, usually composed of an intensive care unit (ICU) physician, an experienced nurse and occasionally a respiratory therapist, which serves to identify and respond to deteriorating patients outside of the ICU. These teams are called rapid response teams (RRTs) or medical emergency teams (METs).

Several before and after studies in Australia, Asia, and the USA (5-7) have proven that there is a significant reduction in adverse events after the implementation of RRTs. Hence, some Chinese hospitals have also recently started to form their own RRTs (8). Nevertheless, no data are available as to the effectiveness of the introduction of RRTs on adverse outcomes for Chinese patients in a Chinese hospital. We hypothesized that RRTs are also associated with an improved outcome in Chinese hospitals. Thus, we compared the hospital outcomes before and after the implementation of RRTs in our hospital, a tertiary teaching hospital in Eastern China.

\section{Methods}

\section{Study setting}

The study was a retrospective review of all hospitalized patients in Sir Run Run Shaw Hospital (SRRSH, Qingchun Campus), of the Zhejiang University School of medicine. The hospital is a 1,200-bed tertiary teaching hospital in Hangzhou, China. From January 1, 2009 to December 31, 2017, we examined the association of the implementation of RRTs and the patients' outcomes. The institutional review board of SRRSH approved the study protocol and waived the need for a consent form (20200207-31) given it was a retrospective study.

Since its opening in 1994, SRRSH has been closely cooperating with the Loma Linda University in California. The president from Loma Linda University managed SRRSH for the first 10 years and continue to closely cooperate and collaborate with the hospital staff. The establishment and development of the SRRSH involved significant aid from US physicians, and included help with the development of emergency department (9). SRRSH has also received recognition for its patient safety, standardized care, and international values, and in 2006, it became the first public hospital in China to receive accreditation from the Joint Commission International (JCI), a US-based,
World Health Organization-authorized organization for medical quality evaluation. In 2017, SRRSH became the first hospital in China to join the Mayo Clinic Care Network and collaborate with the Mayo Clinic (USA). It has built a wide international collaboration network with overseas institutions like Loma Linda University Medical Center and the Mayo Clinic (USA), Shizuoka General Hospital (Japan), and Cabrini Health (Australia).

\section{Establishment of RRTs and study design}

Since its opening in 1994, a code blue on-call team consisting of an ICU fellow physician, an anesthesia fellow, an ICU and emergency nurse leader, and a respiratory therapist in SRRSH has managed the near-collapse or cardiac arrest patients outside the ICU within 5 minutes from the call of ward physicians. Although the code team provides support for in-hospital cardiac arrest resuscitation, we realized the benefit of the team was greatly weakened because the calls are generally activated for patients with cardiac arrest. Therefore, after Dr. Zhou came back from Cabrini hospital in 2010 as a visiting doctor, the SRRSH prepared to adopt an RRT model with the kind help of Dr. John Reeves and Dr. Jonathan Barrett (Cabrini Hospital, Melbourne, Australia). After optimization of the team structure, criteria, and mechanisms for calling, along with repeated education and training, the RRTs were deployed together with the code team in SRRSH in June 2012.

In the present study, a before and after comparison with historical controls was used to determine the effect of the RRT on hospital-wide mortality at SRRSH. Patients admitted before RRT implementation (January 1, 2008, through December 31, 2011) and admission after RRT implementation (January 1, 2013, through December 31, 2017) were retrospectively evaluated.

\section{Intervention}

Any of the following 9 calling criteria could activate the RRTs by any hospital staff: (I) 4 respiratory and airway indications including: (i) obstructed airway, (ii) any difficulty breathing, (iii) respiratory rate less than 8 or greater than 30 breaths per minute, (iv) oximetry saturation less than $85 \%$ despite high flow oxygen; (II) 3 consciousness indications including: (i) sudden changes of consciousness state or inability to rouse patient, (ii) sudden epilepsy attack, (iii) sudden aphasia; (III) 2 circulatory indications including: (i) low blood pressure with systolic pressure less than 
$90 \mathrm{mmHg}$ or $20 \%$ decrease from the baseline and (ii) heart rate less than 40 or greater than 140 beats per minute. However, similar to other hospitals, staff were explicitly instructed to activate the RRT without hesitation for any appropriate degree of clinical concern without the threat of repudiation or reprisal, regardless of whether the reviewed vital signs criteria were met (10). In contrast to the code blue team, the RRT team composes of a single ICU fellow physician, or sometime with an ICU nurse leader or respiratory therapist according to the judgement of patient's status by the ICU fellow. The team is activated by calling the ICU fellow on duty immediately to the bedside to direct the management of an unstable patient. The assessment, management and the patients' disposition are documented simultaneously. The hospital resuscitation committee performed quarterly reviews of all the documents and gave direct feedback to the involved staff about the use of the RRT.

\section{Data sources and processing}

The primary outcome was hospital-wide all-cause mortality. Secondary outcomes included cardiac arrests outside of the ICU. The hospital administration database and RRT documents were queried to show all of the patients' outcomes and whether they had activated the RRTs/code calls. For each year, the following data elements were extracted: (I) total hospitalized patients, (II) number of RRTs and code activation, (III) number of cardiac arrests, (IV) number of patients admitted for medical reasons, and (V) in-hospital mortality. Rates were expressed per 1,000 admissions. The non-obstetric patients were defined as the total admitted patients minus newborn infants. The number of RRT calls was calculated as the number of ICU consultants minus code blue numbers. After some time, physicians would just call the ICU physicians for consultations but did not document this on the patients' records, this might have caused an underestimation of the number of RRT activations.

\section{Primary data analysis}

The Statistical Package for the Social Sciences (SPSS, Version 20.0, Chicago, IL, USA) was used for the analysis. Categorical variables were compared by chi-square analysis. Continuous variables were presented as the mean value. With respect to the differences in the baseline and hospital outcomes between patients pre and post RRT intervention, categorical variables were compared using chi-square analysis, while continuous variables were compared using an independent sample $t$-test for normally distributed data and Mann-Whitney $U$ test for non-normally distributed data. For the trend analysis of hospital-wide mortality, rates of the activation of RRTs and code blue from 2009 to 2017, a linear-by-linear association test was conducted. The relationship between RRT activation and hospitalwide mortality and code blue rates per the calendar year was examined using a linear regression model. A $\mathrm{P}$ value $<0.05$ was considered statistically significant.

\section{Results}

A total of 149,218 and 353,513 patients, respectively, were included in the pre-RRT period (2009-2011) and postRRT period (2013-2017) (Table 1). The average annual admissions in the pre-RRT period were 49,739 patients, and it increased nearly $40 \%$ in the post-RRT period to around $70,703(\mathrm{P}<0.001)$ (Table 1). Similarly, the average annual operations and ICU admissions were significantly higher in the post-intervention years (40,707 vs. 26,830, $\mathrm{P}=0.001$ and 3073 vs. 2141, $\mathrm{P}=0.002$ respectively) (Table 1). Of the admissions, approximately one-third of both periods were admitted for medical reasons. The annual average patient care days were comparable because only a small number of hospital beds were added.

There were 834 RRT activations between 2013 and 2017 after the implementation of RRT in the middle of 2012, i.e., 2.39 per 1,000 non-obstetric patients and 0.35 per 1,000 patient-days (Table 2). There were 301 and 394 code callings in the pre-RRT and post-RRT periods respectively. There was no significant difference in the average code blue activations in per 1,000 non-obstetric patients or per 1,000 patientdays (Table 2). A total of 416 and 612 patients died during hospitalization in the pre- and post-study periods, respectively. The mortality rate was significantly decreased from 2.87 to 1.76 per 1,000 non-obstetric patients after the RRT intervention ( $\mathrm{P}=0.001$, Table 2). This decreasing trend was consistent with respect to the subgroup analysis of patients admitted for both medical $(\mathrm{P}=0.01)$ and surgical reasons $(\mathrm{P}=0.001)$ (Table 2). Among the in-hospital deaths, one-third happened outside the ICU, while approximate $10 \%$ was within $48 \mathrm{~h}$ after admission in both periods ( $\mathrm{P}>0.05$, Table 2$)$.

Although the hospital mortality had remained stable around 3.0 per 1,000 patients from 2009-2011 before the introduction of RRT, there was a significant decrease of hospital mortality from 2.95 to 1.77 per 1,000 non-obstetric 
Table 1 Demographic characteristics of Pre-RRT and Post-RRT intervention patients

\begin{tabular}{lccc}
\hline Variables & Pre-RRT (2009-2011) & Post-RRT (2013-2017) & P value \\
\hline Total admissions & 149,218 & 353,513 & $36.1 \pm 3.2$ \\
Medical admissions (\%) & $37.1 \pm 1.9$ & $70,703 \pm 2,389$ & $<.664$ \\
Admissions/year & $49,739 \pm 5,579$ & $69,737 \pm 2,251$ & $<0.001$ \\
Average non-obstetric admissions/year & $48,224 \pm 5,224$ & $40,707 \pm 3,140$ & 0.001 \\
No. of operations/year & $26,830 \pm 3,375$ & $3,073 \pm 183$ & 0.002 \\
ICU admissions/year & $2,141 \pm 355$ & $472,383 \pm 4,739$ & 0.074 \\
Average annual patient days/year & $423,207 \pm 25,132$ & &
\end{tabular}

$\mathrm{RRT}$, rapid response team; ICU, intensive care unit.

Table 2 Comparison of the hospital outcomes before and after implementation of RRT

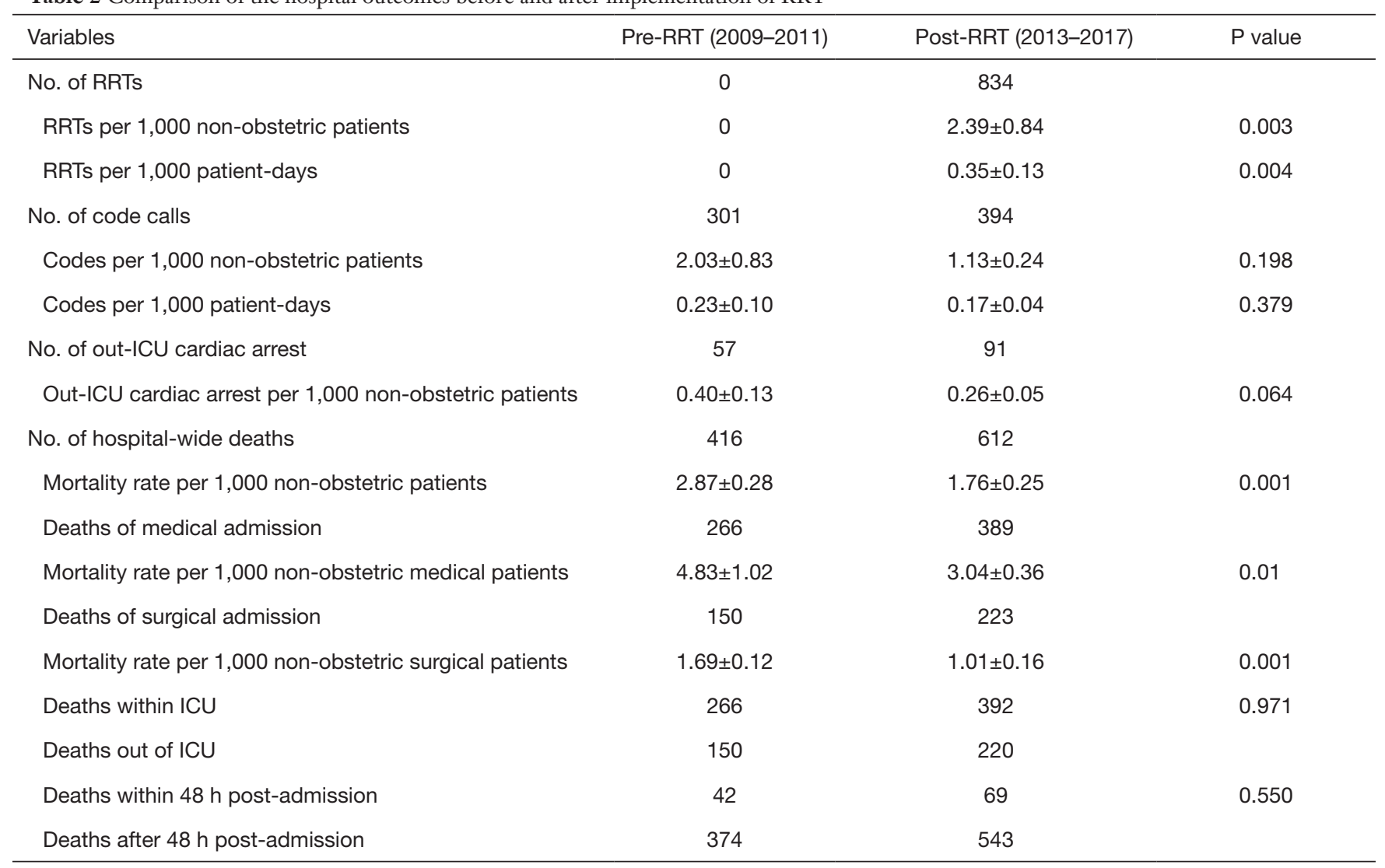

$R R T$, rapid response team; ICU, intensive care unit.

patients after the implementation of RRT $(\mathrm{P}=0.001)$ and the annual mortality decreased constantly $(\mathrm{P}=0.037$ for the trend, Table 3, Figure 1). Moreover, the increase of RRT activations was significantly correlated with the decrease of hospital-wide mortality $(\mathrm{P}=0.025)$ but not correlated with the code blue rate $(\mathrm{P}=0.171)$ (Table 3, Figure 1).

\section{Discussion}

\section{Summary of major findings}

Although some hospitals in China have implemented a medical emergency or rapid response team recently, no data was available on the effect of RRTs on hospital mortality 
Table 3 Trends of annual mortality and rates of Code blue and RRTs activation from 2009 to 2017

\begin{tabular}{|c|c|c|c|c|c|c|c|c|c|}
\hline Rates & 2009 & 2010 & 2011 & 2012 & 2013 & 2014 & 2015 & 2016 & 2017 \\
\hline Code blue activation $/ 1,000^{*}$ & 1.07 & 2.50 & 2.52 & 1.52 & 0.75 & 1.02 & 1.25 & 1.28 & 1.36 \\
\hline RRTs activation $/ 1,000^{*}$ & 0.00 & 0.00 & 0.00 & 1.44 & 1.42 & 1.61 & 2.66 & 2.85 & 3.39 \\
\hline Out-ICU Cardiac arrest/1,000* & 0.51 & 0.44 & 0.26 & 0.32 & 0.25 & 0.30 & 0.19 & 0.32 & 0.25 \\
\hline ICU admission percentage (\%) & 4.57 & 3.76 & 4.58 & 4.92 & 4.32 & 4.59 & 4.18 & 4.19 & 4.45 \\
\hline
\end{tabular}

${ }^{*}$, per 1,000 non-obstetric patients. RRT, rapid response team; ICU, intensive care unit.

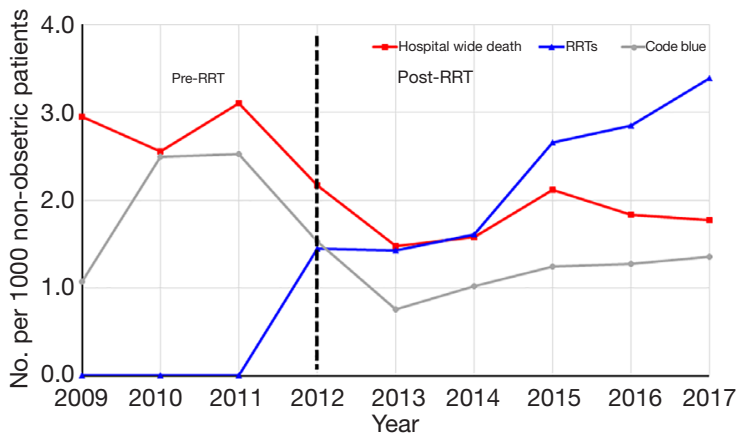

Figure 1 Trends of annual mortality, rates of code blue, and RRTs activation per 1,000 non-obstetric patients from 2009 to 2017. The RRTs were introduced in the middle of 2012 after months of preparation. Therefore, the preintervention period was from 2009 to 2011, while the postintervention period was from 2013 to 2017. $\mathrm{P}=0.037$ for the trend of hospital-wide mortality; $\mathrm{P}=0.025$ for the correlation between the hospital-wide mortality and RRT activations per 1,000 non-obstetric patients.

rate. In our detailed study of a Chinese tertiary hospital over 9 years, involving over 550,000 patients, we demonstrated that the RRT was significantly associated with decreased hospital mortality both in surgical and medical patients. However, different from earlier studies, the increase of RRT activation was not correlated with the decrease of code calls.

\section{Comparison with previous studies}

Several earlier studies have shown reduced mortality after the introduction of a MET (11-13). Chen et al. found that the RRT uptake increased from $32 \%$ to $74 \%$ and was associated with a $52 \%$ decrease in the rate of in-hospital cardiac arrests, a $55 \%$ decrease in cardiac arrest related death, a $23 \%$ decrease in hospital mortality rate, and a
$15 \%$ increase in survival to discharge after in-hospital cardiac arrest (14). Therefore, the National Patient Safety Goals of the Joint Commission states that hospitals should implement a plan to improve the recognition of and response to deteriorating patients. Likewise, the Institute of Healthcare Improvement recommends implementing an early intervention plan for deteriorating patients, such as a rapid response system, as one of their 6 strategies for their 100,000 Lives Campaign (15). However, the abovementioned studies have been criticized for the use of historical controls and were therefore were followed by a large multicentered cluster-randomized trials (MERIT) that was unable to demonstrate a significant effect on a composite outcomes of cardiac arrest, hospital mortality, and unexpected ICU admission in hospitals randomized to implement an MET (16). There were some possible reasons to explain why the MERIT study failed to demonstrate the expected effectiveness of a MET. First, the preimplementation education period was only 3 months, which may be insufficient training time and intensity as the cultivation of safety culture in hospital staff takes time and constant review (5). Another reason for the failure of the MERIT study was that it evaluated the outcomes from the period 6 months after the implementation of the MET in 23 hospitals in Australia, this time interval may also be too short as the change of safety culture also takes time. Lastly, around the time of the MERIT study, approximate $60 \%$ of hospitals had incorporated the MET into practice in Australia and New Zealand. Interestingly, 5 of 11 control hospitals in the MERIT study had introduced a MET system, and that also exhibited a temporal trend toward reduced cardiac arrests (17). As a result, MET activated rates in the experimental arm in the MERIT study were 8.7/1,000 admissions while they were $3.1 / 1,000$ in the control arm (16). Thus, with this "contaminated" control hospitals may underestimate the 
benefit of MET to prevent adverse events. Moreover, the implementation of RRT/MET affected the disposition of the patients with cardiac arrest. After introducing a national framework including a mandatory rapid response system to improve the care of deteriorating patients, cardiac arrestrelated ICU admissions from the ward decreased from 5.6\% (baseline) to $4.9 \%$ (rollout) and $4.1 \%$ (intervention period) in Australia and New Zealand hospitals (2). In our study, although the all-cause hospital mortality remained at an approximate 3.0/1,000 patients for at least 3 years before the implementation of RRT in 2012, it decreased consequently and consistently with the implementation of RRT.

The success of RRTs has led to a belief that increased calling rates may lead to a more reduced mortality rate (18). Devita et al. (19) showed that an increase in the calling rate from $13.7 / 1,000$ to $25.8 / 1,000$ was associated with a reduction in cardiac arrest rates from $6.5 / 1,000$ to $5.4 / 1,000$. Hence, to optimize the benefit of RRT and to decrease preventable in-hospital cardiac arrest, hospitals are encouraged to attain rates of 30 calls/1,000 admissions (20). In contrast to this suggested RRT calling dosage, the RRT activation rate in our hospital increased to an approximate 3.0 calls/1,000 admissions, which was far lower than reported. Three reasons may explain this large discrepancy. The first is that some consultations for the deteriorating patients on the wards were contacted by the ward doctors directly and this was not documented on the charts, and therefore cannot be extracted by the retrospective study. Meanwhile, there were 62 ICU beds in the study hospital, and critically ill patients from clinics or emergency department are given priority and encouraged to be assigned to the ICU by the hospital. This policy may greatly decrease the number of borderline moderate sick patients being disposed to the ward and subsequently decrease the number of potential RRT calling candidates. Importantly, some critically ill patients choose to leave the hospital or sign the "do not resuscitate" order rather than calling the RRT team when their situation meets the RRT criteria as the vast majority of Chinese people prefer to die at their homes. Nevertheless, Santamaria et al. examined 3,339,789 discharges from 15 Victorian hospitals in Melbourne and found that the adjusted mortality was not reduced by higher calling rates in the year of discharge (odds ratio, 1.003; 95\% CI, 1.001-1.006) even when emergency calling rates increased to as high as a median 25.75 per 1,000 discharges (18). An extremely high rate of RRT calling may occur either due to too many critically ill patients in the general wards or due to some patients having multiple
MET reviews before the limitation of medical treatment orders. Occasionally, an increasing number of calls for patients who do not meet usual calling criteria might reflect the difficulties ward staff have in contacting members of the treating team who may be in operating theaters or in outpatient clinics (18). In a 1-month study of 518 patients in 7 Australian hospitals, Jones et al. (21) showed that $10.8 \%$ had limitations of medical treatment orders after the emergency call. Hence, a very high calling of RRTs does not necessarily imply progressively lower adverse events, and may instead lead to a saturation phenomenon.

Besides the RRT calling dose, the time for evaluating the implementation of RRT has been closely associated with the effectiveness of RRTs. Kenward et al. documented the unchanged hospital mortality (20 per 1,000 admissions vs. 19.7 per 1,000 admissions) and cardiac arrest rates (2.6 per 1,000 admissions and 2.4 per 1,000 admissions) 12 months after implementation of MET (22). However, Santamaria et al. (5) found cardiac arrests consistently decreased over the 4.5 years after implementation, and even the longterm total hospital mortality had remained stable for 10 years before the introduction of a MET. This ongoing reduction in cardiac arrests and mortality may be due to a learning effect within the institution and it may take some years to engender a culture change for general ward staff to call the team to critically ill patients (5). Jones et al. also documented reductions in cardiac arrest rates from 4.06 per 1,000 admissions before MET to 1.3 per 1,000 admissions in 4 years after implementation (23).

In the MERIT study, the primary outcome was the composite number of cardiac arrests, unexpected deaths, or unplanned ICU admissions during the 6-month study period after MET activation. In our study, the code rates were not significantly different after the implementation of an RRT ( 0.23 vs. 0.17 per 1,000 patient days, $\mathrm{P}=0.379$ ). This may be due to the preexisting in-house code teams (since the opening of the SRRSH) before RRT implementation. With 24-h/7-d in-house physicians (residents and fellows on duty in hospital, with attending on call for backup) coverage for hospitalized patients as well as along with a 24-h/7-d code team, if a nurse or other staff believes a patient is deteriorating, a physician and sometime an ICU physician of the code team is always available to evaluate the patient, which is actually an RRT-like practice. This may have diminished the value of the "true" RRT since 2012. This was consistent with the study in an American academic hospital with 24-h in-house resident coverage. Although there was a small but non-sustained decrease in overall 
hospital mortality after the implementation of RRT. Khah and colleagues (15) found that RRT implementation did not reduce code rates in the 27 months after intervention ( 0.83 vs. 0.98 per 1,000 patient days, $\mathrm{P}=0.30$ ). Moreover, the frequency of cardiac arrests in our study was $0.32 / 1,000$ admissions. This is far lower than some Australian studies which report a frequency of cardiac arrests between 1.46 and 3.76/1,000 admissions in the context of a medical emergency team (24). This may also be consistent with the fact that some critically ill patients prefer to leave the hospital and to die at home when their situation becomes critical rather than wait to be resuscitated.

\section{Study strengths and limitations}

Successfully implementing an RRT/MET is time-consuming and expensive, it should be initiated in a format that can be adapted to each hospital's specific environment (6). Nevertheless, hospitals are widely implementing RRTs despite uncertainty about their effectiveness. The presence of METs within an institution has been promoted as a legitimate indicator of quality care in Australia and New Zealand (17). For the first time, our study showed that the RRT also improved the overall hospital mortality rates for patients in a tertiary teaching hospital in Eastern China.

Despite the above features, the study is retrospective and observational, and has all the associated limitations. We used the administration data which provided the overall data of annual admissions and discharges, but data for every patient was not available. Thus, we cannot adjust all the cofounding data and provide an odds ratio for the mortality rates. Nevertheless, the percentages of the medical admission and ICU admissions were constant within all the study years. Meanwhile, the decreasing trend of hospital mortality was consistent with respect to the subgroup analysis of patients admitted both for medical and surgical reasons. In addition, our study did not report the incidence of other outcomes, such as unexpected deaths or unplanned ICU admissions, which were used as one of the major composite outcomes for the MERIT study. These data were not recorded prospectively, and we cannot extract the data retrospectively. Last, the overall hospital mortality may include some patients with limitations on medical treatment orders or patients undergoing palliative care.

In conclusion, the implementation of an RRT in a Chinese tertiary hospital was associated with reduced overall hospital mortality both for surgical and medical patients.

\section{Acknowledgments}

We would like to thank Prof. Yun-Xian Yu for statistical assistance, and Ms. Li-Ping Zhou, Ms. Xiang-Ping Chen, Mr. Jin-Ming Ye, Ms Fen Shen and Ms. Qiu-Hua Zhang to provide help in data extraction.

Funding: This work was partly supported by Zhejiang Provincial Basic and Public Welfare Research Program (LGF19H150005).

\section{Footnote}

Conflicts of Interest: The authors have no conflicts of interest to declare.

Ethical Statement: The authors are accountable for all aspects of the work in ensuring that questions related to the accuracy or integrity of any part of the work are appropriately investigated and resolved. Access to the database for research was approved by the Institutional Review Boards of the Sir Run Run Shaw Hospital, Zhejiang University school of medicine.

Open Access Statement: This is an Open Access article distributed in accordance with the Creative Commons Attribution-NonCommercial-NoDerivs 4.0 International License (CC BY-NC-ND 4.0), which permits the noncommercial replication and distribution of the article with the strict proviso that no changes or edits are made and the original work is properly cited (including links to both the formal publication through the relevant DOI and the license). See: https://creativecommons.org/licenses/by-nc-nd/4.0/.

\section{References}

1. Fernando SM, Fox-Robichaud AE, Rochwerg B, et al. Prognostic accuracy of the Hamilton Early Warning Score (HEWS) and the National Early Warning Score 2 (NEWS2) among hospitalized patients assessed by a rapid response team. Crit Care 2019;23:60.

2. Jones D, Bhasale A, Bailey M, et al. Effect of a National Standard for Deteriorating Patients on Intensive Care Admissions Due to Cardiac Arrest in Australia. Crit Care Med 2018;46:586-93.

3. Andersen LW, Holmberg MJ, Berg KM, et al. In-Hospital Cardiac Arrest: A Review. JAMA 2019;321:1200-10.

4. Andersen LW, Kim WY, Chase M, et al. The prevalence and significance of abnormal vital signs prior to in-hospital cardiac arrest. Resuscitation 2016;98:112-7. 
5. Santamaria J, Tobin A, Holmes J. Changing cardiac arrest and hospital mortality rates through a medical emergency team takes time and constant review. Crit Care Med 2010;38:445-50.

6. Kim Y, Lee DS, Min H, et al. Effectiveness Analysis of a Part-Time Rapid Response System During Operation Versus Nonoperation. Crit Care Med 2017;45:e592-9.

7. Foraida MI, DeVita MA, Braithwaite RS, et al. Improving the utilization of medical crisis teams (Condition C) at an urban tertiary care hospital. J Crit Care 2003;18:87-94.

8. Yang $M$, Zhang $\mathrm{L}$, Wang $\mathrm{Y}$, et al. Improving rapid response system performance in a Chinese Joint Commission International Hospital. J Int Med Res 2019;47:2961-9.

9. Clem KJ, Thomas TL, Wang YT, et al. United States physician assistance in development of emergency medicine in Hangzhou, China. Ann Emerg Med 1998;32:86-92.

10. Greiner AL, Stehling-Ariza T, Bugli D, et al. Challenges in Public Health Rapid Response Team Management. Health Secur 2020;18(S1):S8-13.

11. Chan PS, Khalid A, Longmore LS, et al. Hospital-wide code rates and mortality before and after implementation of a rapid response team. JAMA 2008;300:2506-13.

12. Konrad D, Jäderling G, Bell M, et al. Reducing in-hospital cardiac arrests and hospital mortality by introducing a medical emergency team. Intensive Care Med 2010;36:100-6.

13. Lee HY, Lee J, Lee SM, et al. Effect of a rapid response system on code rates and in-hospital mortality in medical wards. Acute Crit Care 2019;34:246-54.

14. Chen J, Ou L, Hillman KM, et al. Cardiopulmonary arrest and mortality trends, and their association with rapid response system expansion. Med J Aust 2014;201:167-70.

15. Shah SK, Cardenas VJ Jr, Kuo YF, et al. Rapid response

Cite this article as: Gong XY, Wang YG, Shao HY, Lan P, Yan RS, Pan KH, Zhou JC. A rapid response team is associated with reduced overall hospital mortality in a Chinese tertiary hospital: a 9-year cohort study. Ann Transl Med 2020;8(6):317. doi: 10.21037/atm.2020.02.147 team in an academic institution: does it make a difference? Chest 2011;139:1361-7.

16. Hillman K, Chen J, Cretikos M, et al. MERIT study investigators: Introduction of the medical emergency team (MET) system: A cluster-randomised controlled trial. Lancet 2005;365:2091-7.

17. Jones D, George C, Hart GK, et al. Introduction of medical emergency teams in Australia and New Zealand: a multi-centre study. Crit Care 2008;12:R46.

18. Santamaria J, Moran J, Reid D. Increasing the Number of Medical Emergency Calls Does Not Improve Hospital Mortality. Crit Care Med 2018;46:1063-9.

19. DeVita MA, Braithwaite RS, Mahidhara R, et al. Medical Emergency Response Improvement Team (MERIT): Use of medical emergency team responses to reduce hospital cardiopulmonary arrests. Qual Saf Health Care 2004;13:251-4.

20. Jones D, Bellomo R, DeVita MA. Effectiveness of the medical emergency team: The importance of dose. Crit Care 2009;13:313.

21. Jones DA, Bagshaw SM, Barrett J, et al. The role of the medical emergency team in end-of-life care: A multicenter, prospective, observational study. Crit Care Med 2012; 40:98-103.

22. Kenward G, Castle N, Hodgetts T, et al. Evaluation of a medical emergency team one year after implementation. Resuscitation 2004;61:257-63.

23. Jones D, Bellomo R, Bates S, et al. Long term effect of a medical emergency team on cardiac arrests in a teaching hospital. Crit Care 2005;9:R808-15.

24. Jones D, Mercer I, Heland M, et al. In-hospital cardiac arrest epidemiology in a mature rapid response system. $\mathrm{Br}$ J Hosp Med (Lond) 2017;78:137-42. 